JOCHEM KAHL

\title{
Die Farbgebung in der frühen Hieroglyphenschrift
}

Ziel der folgenden Untersuchung ist, eine Grundlage für die Beschäftigung mit der Farbgebung frühzeitlicher Hieroglyphen zu erstellen ${ }^{1}$.

Schriftzeichen werden durch einen Kontrast zwischen Schriftträger und Schreibmaterial deutlich gemacht. In der 0.-1. Dynastie wurde dieser Kontrast durch Reliefierung, Einritzung oder Beschriftung mit Tusche erzielt. Bei der Technik der Einritzung konnte eine einfarbige Paste, die in die Vertiefungen des Schriftträgers gefüllt wurde, den Kontrast verstärken. Bei der Beschriftung mit Tusche wurde zumeist allein schwarze Tusche, seltener auch allein rote Tusche verwendet. Gelegentlich sind aber auch Inschriften belegt, die mehrfarbig gestaltet sind. Das derzeit früheste bekannte Beispiel ist eine Öletikette aus Umm el-Qaab, Grab B 18² ; dieses Grab ist nach seinen Funden in die Zeit Narmers zu datieren. Die Öletikette aus Elfenbein trägt eine Inschrift aus schwarz bzw. rot gezeichneten Hieroglyphen. Aus der 1. Dynastie sind 24 weitere Etiketten (zum Teil Jahrestäfelchen) mit Nennung von Ölen, Stoffen, Früchten oder Getreide bekannt, auf denen mit schwarzer und roter Tusche Hieroglyphen geschrieben sind (s. Anhang I). Auf einem Jahrestäfelchen des Djer war die Farbkombination Grün/Rot für die Schreibung der Hieroglyphen gewählt worden, und auf einem Stab aus Elfenbein, der den Namen des Königs „Schlange“" trägt, weisen die Hieroglyphen Reste roter und blauer Farbe ${ }^{3}$ auf (s. Anhang I).

Ursprünglich waren sicherlich mehr Inschriften der 0. und 1. Dynastie in verschiedenen Farben ausgeführt ${ }^{4}$, doch sind die Farben heute wohl oft verschwunden, oder es wurde von den Ausgräbern bzw. den Bearbeitern der Inschriften auf das Feststellen der Färbung kein Wert gelegt ${ }^{5}$.

Während alle 27 dokumentierten farbigen Inschriften der 0.-1. Dynastie zweifarbig sind, sind aus der 2. Dynastie bislang keine zwei- oder mehrfarbigen Inschriften bekannt. Aus der 3. Dynastie liegen jedoch wieder Beispiele für farbige - dieses Mal mehrfarbige - Inschriften vor (s. Anhang II). Auf den Wandmalereien im Grab des hsi.y-r $r^{c}$ (S 2405) und auf den Scheintür-

${ }^{1} \mathrm{Zu}$ Farben in ägyptischer Schrift und Sprache vgl. Smith, Sculpture and Painting, 257-263, 366-382; Schenkel, Farben; B aines, Color Terminology and Color Classification; Sta ehelin, Farben.

${ }^{2}$ Petrie, Royal Tombs II, Taf. 12.4.

${ }^{3}$ So: Emery, Tombs of the First Dynasty II, 104 (Abb. 106); einen noch früheren Beleg für Blau liefert eine Schale aus Hierakonpolis (Thomas, Discovery, 112).

${ }^{4}$ Nach dem erhaltenen Befund dürfte insbesondere bei Etiketten (dazu zählen auch die Jahrestäfelchen) mit Farbigkeit der Hieroglyphen zu rechnen sein.

${ }^{5}$ Vgl. Staehelin, Farben, 102.

${ }^{6}$ Quibell, Hesy. 
nischen aus Kalkstein aus dem Grab des he-b3.w-skr (S 3073) ${ }^{7}$ (mit Hieroglyphen in erhabenem Relief) sind farbige Hieroglyphen erhalten. Im Gegensatz zu den Inschriften der 0. und 1. Dynastie wurden in der 3. Dynastie mehr als zwei Farben auf einem Schriftträger verwendet.

Im Grab des $h h^{r}-b 3 . w-s k r$ wurden die Schriftzeichen auf den Scheintürnischen aus Kalkstein in vier Farben bemalt: in Schwarz, Rot, Gelb und Grün. Der Farbgebrauch wurde von Murray auch gut dokumentiert ${ }^{8}$.

Im Grab des hsi.y-r $r^{\ulcorner} w$ sind im oberen Register der Ostwand des „painted corridor ${ }^{\text {“9 }}$ farbige Hieroglyphen gemalt, die zur Zeit von Quibells Ausgrabung (1911-1912) nur noch schlecht erhalten waren ${ }^{10}$. Zumeist war die oberste, endgültige Farbschicht abgefallen, so daß darunterliegende Schichten freigelegt waren ${ }^{11}$, deren Farbe nicht unbedingt mit derjenigen der obersten Schicht identisch sein mußte. Für eine Auswertung des Farbgebrauchs kommt erschwerend hin$\mathrm{zu}, \mathrm{daß}$ Quibell die erhaltene Farbe der Hieroglyphen nur wenig umfassend dokumentiert hat ${ }^{12}$. Quibell beschränkte sich hauptsächlich auf Schwarz-weiß-Zeichnungen der Inschriften ${ }^{13}$, die lediglich schwarze Hieroglyphen durch ihre schwarze Ausmalung kennzeichnen, jedoch offen lassen, ob es sich bei den Hieroglyphen, die ohne Ausmalung wiedergegeben sind, um andersfarbige handelt, deren Farbe auch noch erkennbar war, oder um solche, deren Farbe zerstört war $^{14}$. Aufgrund der Farbtafel Quibell, Hesy, Taf. 10, der Erwähnung von roten und schwarzen Hieroglyphen ${ }^{15}$ und der in den bildhaften und geometrischen Wandmalereien des „painted corridor" verwendeten Farben Schwarz, Rot, Gelb, Grün, Weiß und Blauschwarz ${ }^{16}$ ist allerdings anzunehmen, daß im Grab des hsi.y-r ${ }^{\complement} w$ mehr als zwei Farben für die Wiedergabe der Schriftzeichen verwendet wurden. Da die Bestimmung der Farben der Hieroglyphen im Einzelfall aber kaum erfolgt ist, konnte im vorliegenden Artikel nur in wenigen Fällen eine Farbzuweisung vorgenommen werden ${ }^{17}$.

Für die Farbgebung der Hieroglyphen ab der Zeit des Alten Reiches konstatierte Staehelin Regelmäßigkeiten, die sie als „Kanon“ bezeichnete", wobei aber durchaus Abweichungen von dieser Norm festzustellen sind ${ }^{19}$. Für die Schriftzeugnisse der 0. und 1. Dynastie sind gleichfalls Normen in der Farbgebung der Hieroglyphen erkennbar, die allerdings von denen, die Staehelin feststellte, teilweise abweichen. Soweit überliefert, sind generell höchstens zwei Farben auf einem Schriftträger verwendet worden, um die Schriftzeichen sichtbar zu machen. Dabei scheint die Kombination Schwarz/Rot die Regel gewesen zu sein, der gemeinsame Gebrauch von Rot und Grün (Qu. 847) bzw. von Rot und Blau (Qu. 987) dagegen die Ausnahme. Eine nähere Untersuchung des Farbgebrauchs in den 25 Inschriften der 0.-1. Dynastie mit roten und schwarzen Hieroglyphen (vgl. Anhang III) erlaubt folgende Aussagen:

${ }^{7}$ Murray, Saqqara Mastabas, 2-4, 32-36, 40-46.

${ }^{8}$ Murray, Saqqara Mastabas, Taf. $41-45$.

${ }^{9}$ Vgl. Quibell, Hesy, Taf. 1.

${ }^{10} \mathrm{Vgl}$. Quibell, Hesy, 18: „In the upper register very little indeed is left, and, except at the south end there is no colour".

${ }^{11}$ Quibell, Hesy, 17: "Much of the colour has fallen away and left different stages of the work visible".

${ }^{12}$ Die Publikation enthält lediglich drei konkrete Farbangaben (Quibell, Hesy, 36) und eine Farbtafel mit Hieroglyphen (Quibell, Hesy, Taf. 10).

${ }^{13} \mathrm{Vgl}$. Quibell, Hesy, Taf. 18-22.

${ }^{14} \mathrm{Zu}$ den Hieroglyphen von Quibell, Hesy, Taf. 17-21 gibt Quibell keine Beschreibung.

${ }^{15}$ Quibell, Hesy, 36 (zu Taf. 22).

${ }^{16}$ Blauschwarz (,,bluish black“", „blue-black“) ist zum Beispiel in der Darstellung einer Axt verwendet (Quibell, Hesy, 21). Zu den anderen Farben vgl. Quibell, Hesy, Taf. 8-9, 11-14.

${ }^{7}$ Selbst eine Farbtafel wie Quibell, Hesy, Taf. 10 gibt keinen Aufschluß darüber, ob zum Beispiel von den Hieroglyphen $\square$ oder $\square$ noch Reste der endgültigen obersten Farbschicht vorhanden waren oder nur Reste der Vorzeichnung.

${ }^{18}$ Staehelin, Farben, 101-102.

${ }^{19} \mathrm{Sta}$ thelin, Farben, 102. 
1. Manche Hieroglyphen wurden einfarbig rot oder schwarz geschrieben, andere zweifarbig schwarz und rot.

2. Alle Hieroglyphen weisen eine relativ verbindliche Farbgebung auf (d. h. einfarbig rot bzw. einfarbig schwarz bzw. zweifarbig schwarz und rot), von der selten abgewichen wurde. Die Farbgebung der einzelnen Hieroglyphen und ihre Belege können dem Anhang III entnommen werden. Im folgenden seien nur einige der Regelmäßigkeiten hervorgehoben: Generell wurde die Hautfarbe des menschlichen Körpers rot wiedergegeben, seine Haare jedoch schwarz (vgl. A 22, A 34, A 49 , a 13, D 1, D 28, D 36, D 46, D 46*, W 25). Manche Vögel wurden schwarz gemalt (G 5, G 17, G 27, G 36), andere rot (G 14, G 43). Wasser wurde regelmäßig durch schwarze Farbe angedeutet (D 46*, N 35, N 37, N 39, p 4; vgl. auch N 40 und N 41 in der 3. Dynastie). Auch Gebäudegrundrisse erschienen schwarz (O 1, O 6; vgl. O 4 in der 3. Dynastie).

Aus der 2. Dynastie sind bislang keine farbigen Inschriften überliefert. In den Inschriften der 3. Dynastie ist die ausschließliche Zweifarbigkeit der Hieroglyphen einer Mehrfarbigkeit (im Falle des $h h^{-}-b 3 . w$-skr einer Vierfarbigkeit) gewichen. Ein Vergleich der Farbgebung der 0.-1. Dynastie mit derjenigen der 3. Dynastie läßt erkennen, daß es sich bei der Farbgebung der 3. Dynastie um eine systematische Weiterentwicklung der schwarz/roten Farbverteilung der 0.-1. Dynastie handelt:

- Die Hieroglyphen, die in den in schwarzer und roter Farbe gehaltenen Inschriften der 0.-1. Dynastie schwarz geschrieben waren, sind auch in der 3. Dynastie schwarz: G 36, I $10^{20}$, M 16 $6^{21}, \mathrm{~N} 35, \mathrm{~N} 37, \mathrm{~W} 24, \mathrm{X} 1$.

- Die Hieroglyphen, die in den in schwarzer und roter Farbe gehaltenen Inschriften der 0.-1. Dynastie rot geschrieben waren, sind in der 3. Dynastie wiederum rot oder aber gelb bzw. grün:

- Weiterhin rot sind in der 3. Dynastie: D 36, m 1, S 29, T 8, U 6, W $2^{22}$.

- Gelb sind in der 3. Dynastie: D 21, D 46, F 9 ${ }^{23}$, G 43, M 3.

- Grün sind in der 3. Dynastie: M $23^{24}$ und die in der 1. Dynastie rot dargestellte Binsenmatte von R 4.

In einer Graphik kann die Entwicklung der Farbgebung der Hieroglyphen von der 0./1. Dynastie bis zur 3. Dynastie folgendermaßen dargestellt werden (die in zweifarbigen Inschriften der 1. Dynastie ebenfalls belegten Farben Grün und Blau bleiben als Ausnahmen unberücksichtigt):
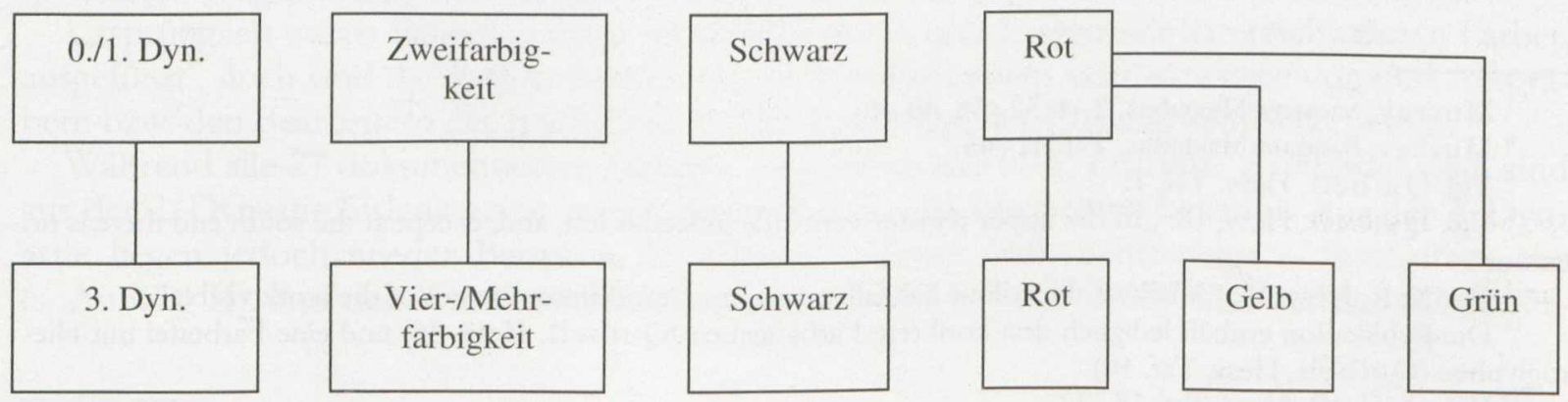

${ }^{20} \mathrm{I} 10$ ist in der 1. Dynastie schwarz, aber auch rot und schwarz belegt.

${ }^{21}$ Im Grab des $h^{c}$-b3.w-skr ist der Erdhügel schwarz, zu dem oberen Teil der Hieroglyphe liegen keine Angaben vor (vgl. Murray, Saqqara Mastabas, Taf. 42). bar.

${ }^{22} \mathrm{Im}$ Grab des hsi.y-r ${ }^{\complement} w$ ist der obere Teil rot und weiß gesprenkelt, die Farbe des unteren Teils nicht bestimm-

${ }^{23}$ Gelb mit schwarzen Flecken.

${ }^{24}$ M 23 wurde in der 1. Dynastie dreimal rot wiedergegeben und einmal abweichend schwarz. 
Obgleich nicht einmal 1\% der derzeit erhaltenen und publizierten Schriftzeugnisse der Frühzeit $^{25}$ Reste von zwei- oder mehrfarbiger Bemalung aufweisen, erlauben die Ergebnisse der Untersuchung ihrer Farbgebung von frühzeitlichen Farbregeln zu sprechen. Diese Farbregeln zeichnen sich durch eine konstante Farbgebung für die einzelnen Schriftzeichen aus, von der nur in Ausnahmefällen abgewichen wurde.

Die regelhafte Weiterentwicklung des zweifarbigen Farbgebrauchs zu einem mehrfarbigen Farbgebrauch, die nach der derzeitigen Quellenlage in der 3. Dynastie stattfand, könnte im Zusammenhang mit anderen Neuerungen bzw. Entwicklungen stehen, die im Schriftsystem der 3. Dynastie zu beobachten sind ${ }^{26}$.

\section{ANHANG I}

Farbige Inschriften der 0-1. Dynastie (die Quellen-Nummern entsprechen denjenigen aus Kahl, System, $169-417)$

Rot-schwarze Inschriften:

Qu. 96: Petrie, Royal Tombs II, Taf. 12.4 (Abydos, Umm el-Qaab, Grab B 18; z. Zt. des Narmer). Qu. 645: Petrie, Royal Tombs II, Taf. 12.1 (Abydos, Umm el-Qaab, Grab O; z. Zt. des Djer).

Qu. 646: Petrie, Royal Tombs II, Taf. 12.3 (Abydos, Umm el-Qaab, Grab O; Djer).

Qu. 834: Quibell, Archaic Mastabas, Taf. 11.5; Kaplony, Inschriften Supplement, Abb. 1064 (Saqqara, Grab S $2171 \mathrm{H}$; Djer).

Qu. 989a-b: Emery, Tombs of the First Dynasty II, Abb. 108 (Saqqara, Grab S 3504; z. Zt. des „,Schlange“).

Qu. 990: Emery, Tombs of the First Dynasty II, Abb. 109 (Saqqara, Grab S 3504; z. Zt. des „Schlange").

Qu. 991: Emery, Tombs of the First Dynasty II, Abb. 110 (Saqqara, Grab S 3504; z. Zt. des „Schlange“).

Qu. 992: Emery, Tombs of the First Dynasty II, Abb. 111 (Saqqara, Grab S 3504; z. Zt. des „Schlange“).

Qu. 993: Emery, Tombs of the First Dynasty II, Abb. 112 (Saqqara, Grab S 3504; z. Zt. des „Schlange“).

Qu. 994: Emery, Tombs of the First Dynasty II, Abb. 113 (Saqqara, Grab S 3504; z. Zt. des „Schlange“).

Qu. 995: Emery, Tombs of the First Dynasty II, Abb. 114 (Saqqara, Grab S 3504; z. Zt. des „Schlange“).

Qu. 1117: Emery, Tombs of the First Dynasty II, Abb. 115 (Saqqara, Grab S 3504; z. Zt. von „Schlange“ - Kaa).

Qu. 1118: Emery, Tombs of the First Dynasty II, Abb. 116 (Saqqara, Grab S 3504; z. Zt. von "Schlange“ - Kaa).

Qu. 1119: Emery, Tombs of the First Dynasty II, Abb. 117 (Saqqara, Grab S 3504; z. Zt. von „Schlange“ - Kaa).

Qu. 1120: Emery, Tombs of the First Dynasty II, Abb. 118 (Saqqara, Grab S 3504; z. Zt. von „Schlange“ - Kaa).

Qu. 1121: Emery, Tombs of the First Dynasty II, Abb. 119 (Saqqara, Grab S 3504; z. Zt. von „Schlange“- Kaa).

Qu. 1122: Emery, Tombs of the First Dynasty II, Abb. 120 (Saqqara, Grab S 3504; z. Zt. von "Schlange“ - Kaa).

Qu. 1123: Emery, Tombs of the First Dynasty II, Abb. 121 (Saqqara, Grab S 3504; z. Zt. von „Schlange“ - Kaa).

Qu. 1124: Emery, Tombs of the First Dynasty II, Abb. 122 (Saqqara, Grab S 3504; z. Zt. von "Schlange“ - Kaa).

Qu. 1125: Emery, Tombs of the First Dynasty II, Abb. 124 (Saqqara, Grab S 3504; z. Zt. von „Schlange“ - Kaa).

Qu. 1126: Emery, Tombs of the First Dynasty II, Abb. 125 (Saqqara, Grab S 3504; z. Zt. von „Schlange“ - Kaa).

Qu. 1390: Godron, Horus Den, Taf. 3.6 (Abydos, Umm el-Qaab; Den).

Qu. 1564: Emery, Tombs of the First Dynasty I, Abb. 65 (Saqqara, Grab X; z. Zt. von Den - Ende 1. Dynastie).

Qu. 1677: Petrie, Royal Tombs I, Taf. 17.26 (Abydos, Umm el-Qaab, Grab U; Semerchet).

Qu. 1931: Emery, Tombs of the First Dynasty II, Abb. 123 (Saqqara, Grab S 3504; Kaa).

Rot-grüne Inschriften:

Qu. 847: Emery, Hemaka, Taf. 18 A (Saqqara, Grab S 3035; Djer).

Rot-blaue Inschriften:

Qu. 987: Emery, Tombs of the First Dynasty II, Abb. 106 (Saqqara, Grab S 3504; ,Schlange“).

${ }^{25}$ Eine Übersicht über die erhaltenen Schriftzeugnisse der 0.-3. Dynastie gibt Kahl, System, 169-417.

${ }^{26} \mathrm{Vgl}$. Kahl, System, 161-162. 


\section{ANHANG II} 417).

Farbige Inschriften der 3. Dynastie (die Quellen-Nummern entsprechen denjenigen aus Kahl, System, 169-

Qu. 3264: Quibell, Hesy, Taf. 10, 15, 17-22 (Saqqara, Grab S 2405; z. Zt. des Netjerichet (Djoser)).

Qu. 3371: Murray, Saqqara Mastabas I, Taf. 1 (Saqqara, Grab S 3073; Ende 3. Dynastie).

Qu. 3372: Murray, Saqqara Mastabas I, Taf. 2 (Saqqara, Grab S 3073; Ende 3. Dynastie).

\section{ANHANG III}

Die Farbgebung der Hieroglyphen in den rot-schwarzen Inschriften der 0.-1. Dynastie und in den Inschriften der 3. Dynastie (Zeichen-Nummern, die aus Großbuchstaben und Ziffern bestehen, entsprechen denen der Zeichenliste von Gardiner, Egyptian Grammar, 438-548; Zeichen-Nummern, die aus Kleinbuchstaben und Ziffern bestehen, entsprechen denjenigen der Zeichenliste von Kahl, System, 419-906; zu den Quellen-Nummern vgl. Anhang I und II).

Abkürzungserklärung: schw = schwarz; $v$ = paläographische Variante des Zeichens

\begin{tabular}{|c|c|c|c|c|c|c|c|c|}
\hline \multirow[b]{2}{*}{$\begin{array}{c}\text { Zeichen- } \\
\text { Nr. }\end{array}$} & \multirow[b]{2}{*}{ Zeichen } & \multicolumn{3}{|c|}{$0 . / 1$. Dyn. } & \multicolumn{4}{|c|}{ 3. Dyn. } \\
\hline & & rot & schw & $\begin{array}{l}\text { rotu } \\
\text { schw }\end{array}$ & rot & gelb & grün & schw \\
\hline A 22 (?) & 算 & & & $1564^{27}$ & & & & \\
\hline A 34 & 乩 & & & $1390^{28}$ & & & & \\
\hline A $49^{\mathrm{V}}$ & กी & & & $1390^{29}$ & & & & \\
\hline a 13 & 务 & & & $1677^{30}$ & & & & \\
\hline D 1 & 2) & & & $\begin{array}{l}989 a^{31} \\
990^{32} \\
994^{33}\end{array}$ & & & & \\
\hline D 4 & $\infty$ & & & & & & & $3371-2$ \\
\hline D 19 & B & 1390 & & & & & & \\
\hline D 21 & 0 & 1390 & & & & 3372 & & \\
\hline D 28 & $U$ & $\begin{array}{l}646 \\
989 a \\
990-1 \\
993-5 \\
1390 \\
1564 \\
1677 \\
\end{array}$ & & & & & & \\
\hline
\end{tabular}

${ }^{27}$ Rote Hautfarbe, schwarze Perücke, schwarzer waagerechter Stab.

${ }^{28}$ Rote Hautfarbe, schwarze Perücke, schwarzer Schurz, schwarzer Bottich.

29 Rote Hautfarbe, schwarze Haare, schwarzer Bart.

${ }^{30}$ Rote Hautfarbe, schwarze Perücke, schwarzer Schurz.

${ }^{31}$ Rote Hautfarbe, schwarze Perücke, schwarzer Bart.

${ }^{32} \mathrm{~S}$. vorige Anm.

${ }^{33} \mathrm{~S}$. vorige Anm. 


\begin{tabular}{|c|c|c|c|c|c|c|c|c|}
\hline \multirow[b]{2}{*}{$\begin{array}{c}\text { Zeichen- } \\
\text { Nr. }\end{array}$} & \multirow[b]{2}{*}{ Zeichen } & \multicolumn{3}{|c|}{ 0./1. Dyn. } & \multicolumn{4}{|c|}{ 3. Dyn. } \\
\hline & & rot & schw & $\begin{array}{l}\text { rotu } \\
\text { schw }\end{array}$ & rot & gelb & grün & schw \\
\hline D 36 & $\longrightarrow$ & $\begin{array}{l}1390 \\
1931 \\
\end{array}$ & & & 3371 & & & \\
\hline D 46 & $\Longrightarrow$ & 1390 & & & & 3372 & & \\
\hline D $46^{*}$ & $\Leftrightarrow$ & & & $\begin{array}{l}645^{34} \\
1677^{35}\end{array}$ & & & & \\
\hline D 54 & $\Lambda$ & $\begin{array}{l}\text { vgl. } \\
\text { W } 25\end{array}$ & & & & & & \\
\hline D 58 & 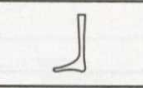 & & & & & $3371-2$ & & \\
\hline E 8 & $x^{2} \times x$ & & & & & & & 3372 \\
\hline e 21 & 诲 & & & $1677^{36}$ & & & & \\
\hline F 4 & (2) & & $\begin{array}{l}96 \\
645 \\
834 \\
989 \mathrm{a} \\
990 \\
994 \\
1117-24 \\
1390 \\
1677 \\
1931 \\
\end{array}$ & & & & & \\
\hline F 9 & ? & 1564 & & & & $\begin{array}{l}3371- \\
3372^{37} \\
\end{array}$ & & \\
\hline F 13 & $\mathbb{U}$ & & 1390 & & & & & \\
\hline F 26 & $5 \pi$ & & & & 3371 & & & \\
\hline F 29 & - $T^{\circ}$ & 1390 & & & & & & \\
\hline F 34 & రิ & & & & 3371 & & & \\
\hline F 35 & t & & & & 3372 & & & \\
\hline F 46 & $\Longleftarrow$ & & & & 3372 & & & \\
\hline f 9 & $\beta$ & & 1390 & & & & & \\
\hline G 1 & A & & & & & $3372^{38}$ & & $3371^{39}$ \\
\hline
\end{tabular}

${ }^{34}$ Rote Hand, schwarze Wassertropfen.

${ }^{35} \mathrm{~S}$. vorige Anm.

${ }^{36}$ Schwarzer Affe, roter Sitz, rote Kugeln.

${ }^{37}$ Gelb mit schwarzen Flecken.

${ }^{38}$ Mit schwarzem Kopf und schwarzen Krallen.

${ }^{39}$ Mit rotem Kopf und roten Krallen. 


\begin{tabular}{|c|c|c|c|c|c|c|c|c|}
\hline & & & 0./1. Dyn. & & & & & \\
\hline $\begin{array}{c}\text { Zeichen- } \\
\text { Nr. }\end{array}$ & Zeichen & rot & schw & $\begin{array}{l}\text { rotu } \\
\text { schw }\end{array}$ & rot & gelb & grün & schw \\
\hline G 5 & Ind & & $\begin{array}{l}646 \\
834 \\
1390 \\
1677 \\
1931 \\
\text { vgl. p } 10\end{array}$ & & & & & \\
\hline G 14 & Ans & $\begin{array}{l}\text { vgl. } \\
\text { G } 16\end{array}$ & & & & & & \\
\hline G 16 & A) & & & $1677^{40}$ & & & & \\
\hline G 17 & ou & & $\begin{array}{l}1126 \\
1390\end{array}$ & & & & & \\
\hline G 27 & N & & 1125 & & & & & \\
\hline G 36 & N & & 1677 & & & & & $3371-2$ \\
\hline G 43 & B & 645 & & & & 3372 & & \\
\hline I 10 & 2 & & 646 & $\begin{array}{l}989 a^{41} \\
990- \\
995^{42}\end{array}$ & & & & 3372 \\
\hline I 12 & 3 & $\begin{array}{l}\text { vgl. } \\
\text { G } 16 \\
\end{array}$ & & & & & & \\
\hline $\mathrm{k} 6$ & cos & $990^{43}$ & & & & & & \\
\hline L 2 & 象 & & & $\begin{array}{l}1677^{44} \\
1690^{45} \\
\end{array}$ & & & & \\
\hline M 3 & Dי & $\begin{array}{l}834 \\
989 \mathrm{a} \\
1117 \\
1124 \\
1677 \\
\end{array}$ & & & & 3372 & & \\
\hline M 4 & $\oint$ & & $\begin{array}{l}1125 \\
1390 \\
1677 \\
\end{array}$ & & & & & \\
\hline M 8 & 80909 & & & & & & & $\begin{array}{l}3371- \\
3372^{46} \\
\end{array}$ \\
\hline
\end{tabular}

${ }^{40}$ Rote Kobra, rote Körbe, schwarzer Geier.

${ }^{41}$ Schwarzer Kopf und schwarzer Fleck auf der Brust, Rest der Kobra rot.

${ }^{42}$ S. vorige Anm.

${ }^{43}$ In Qu. 989a, 992, 994 ist lediglich ein schwarzer Umriß gezeichnet; eine Innenzeichnung fehlt.

${ }^{44}$ Rote Flügel, rote Beine; Körper rot und schwarz.

${ }^{45}$ S. vorige Anm.

${ }^{46}$ Der Teich ist mit schwarzer Farbe gemalt. Zu der Farbe der Lotuspflanze gibt es keine Angabe (vgl. Murray, Saqqara Mastabas, Taf. 43). 


\begin{tabular}{|c|c|c|c|c|c|c|c|c|}
\hline & & & 0./1. Dyn. & & & & & \\
\hline $\begin{array}{c}\text { Zeichen- } \\
\text { Nr. }\end{array}$ & Zeichen & rot & schw & $\begin{array}{l}\text { rotu } \\
\text { schw }\end{array}$ & rot & gelb & grün & schw \\
\hline M 12 & d & & & & & & 3372 & \\
\hline M 16 & 8 & & 834 & & & & & $3372^{47}$ \\
\hline M 23 & $\gamma \psi$ & $\begin{array}{l}1390 \\
1564 \\
1677\end{array}$ & 1390 & & & & 3372 & \\
\hline M 26 & 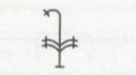 & 1564 & & & & & & \\
\hline M 37 & 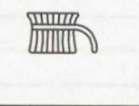 & $\begin{array}{l}646 \\
834\end{array}$ & & & & & & \\
\hline M 41 (?) & $a$ & & & 1677 & & & & \\
\hline $\mathrm{m} 1$ & 0 & 1125 & & & 3372 & & & \\
\hline $\mathrm{m} 2$ & 0 & 1125 & $\begin{array}{l}\text { vgl. m } 2 \\
\text { (vielfach) } \\
u \mathrm{~V} 12^{\text {v48 }}\end{array}$ & & & & & \\
\hline $\mathrm{m} 13$ & 需 & & & $\begin{array}{l}990^{49} \\
994^{50} \\
1118^{51} \\
1931^{52}\end{array}$ & & & & \\
\hline $\mathrm{m} 16$ & $\nabla$ & & & $995^{53}$ & & & & \\
\hline N 11 & $\curvearrowright$ & $\begin{array}{l}1125 \\
1564\end{array}$ & & & & & & \\
\hline $\begin{array}{l}\text { N 20/ } \\
\text { N } 22 \text { (?) }\end{array}$ & $\varpi /$ & & & 1564 & & & & \\
\hline N 25 & $M$ & & & $1390^{54}$ & & & & \\
\hline N 29 & $\Delta$ & & 1931 & & & & & \\
\hline N 35 & mим & & $\begin{array}{l}1125 \\
1390\end{array}$ & & & & & $\begin{array}{l}3264^{55} \\
3371-2\end{array}$ \\
\hline
\end{tabular}

${ }^{47}$ Der Erdhaufen ist mit schwarzer Farbe gemalt; weitere Angaben zur Farbgebung liegen nicht vor (vgl. Murray, Saqqara Mastabas, Taf. 42).

${ }_{48}^{4}$ Die schwarze Farbe läßt vermuten, daß es sich nicht um $\mathrm{m} 2$ handelt, sondern um ein von $\mathrm{m} 2$ verschiedenes Zeichen (speziell: Feigen).

\footnotetext{
${ }^{49}$ Roter Stengel, rote Blätter, schwarze Wurzeln, schwarze Blüten.

${ }^{50} \mathrm{~S}$. vorige Anm.

${ }^{51}$ Vgl. vorige Anm.; eventuell handelt es sich auch um M 23 oder M 26.

${ }^{52}$ S. vorige Anm.

${ }^{53}$ Roter Stiel.

${ }^{54}$ Schwarze Grundlinie, rote Hügel.

${ }^{55}$ Quibell, Hesy, Taf. 21.
} 


\begin{tabular}{|c|c|c|c|c|c|c|c|c|}
\hline & & & 0./1. Dyn & & & & & \\
\hline $\begin{array}{c}\text { Zeichen- } \\
\text { Nr. }\end{array}$ & Zeichen & rot & schw & $\begin{array}{l}\text { rotu } \\
\text { schw }\end{array}$ & rot & gelb & grün & schw \\
\hline N 37 & $\square$ & & 1677 & & & & & $\begin{array}{l}3264^{56} \\
3371-2 \\
\text { vgl. } \\
\text { N } 40\end{array}$ \\
\hline N 39 & 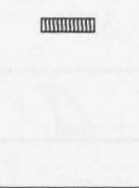 & & $\begin{array}{l}1124^{57} \\
1125 \\
1677 \\
\text { vgl. p } 4\end{array}$ & & & & & \\
\hline N 40 & $\triangle$ & & & & & & & $3264^{58}$ \\
\hline N 41 & Y & & & & & & & 3372 \\
\hline n 3 & $\because$ & & $\begin{array}{l}\text { vgl. } \\
\text { D } 46^{*}\end{array}$ & & & & & \\
\hline O 1 & $\square$ & & $\begin{array}{l}1117-22 \\
1125 \\
1931 \\
\end{array}$ & & & & & \\
\hline O 4 & 口 & & & & & & & 3372 \\
\hline O 6 & ந & & $\begin{array}{l}646 \\
1390\end{array}$ & & & & & \\
\hline O 11 & 狊 & $1677^{59}$ & & & & & & \\
\hline O 28 & 光 & 1390 & & & & & & \\
\hline O 29 & $\infty$ & & & & 3372 & & & \\
\hline O 31 & 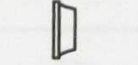 & 1390 & & & & & & \\
\hline O 33 & 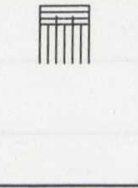 & & $\begin{array}{l}646 \\
834 \\
1390 \\
1931 \\
\end{array}$ & & & & & \\
\hline O 51 & : & & & & & & & $3371-2$ \\
\hline p 4 & 选影 & & & $1677^{60}$ & & & & \\
\hline $\mathrm{p} 10$ & All & & & $1677^{61}$ & & & & \\
\hline p 11 & $\Omega$ & & & & $3264^{62}$ & & & \\
\hline
\end{tabular}

${ }^{56}$ Quibell, Hesy, Taf. 10.

57 Ob es sich um N 39 handelt, ist unsicher.

58 Der Teich ist schwarz; vgl. Quibell, Hesy, Taf. 20.

59 Nur die Umrißzeichnung ist schwarz.

60 Schwarzes Wasser, schwarzer Schiffsrumpf, rote Aufbauten.

61 Schwarzer Falke, schwarzer Schiffsrumpf, rote Bugzier.

${ }^{62}$ Quibell, Hesy, 36. 


\begin{tabular}{|c|c|c|c|c|c|c|c|c|}
\hline \multirow[b]{2}{*}{$\begin{array}{c}\text { Zeichen- } \\
\text { Nr. }\end{array}$} & \multirow[b]{2}{*}{ Zeichen } & \multicolumn{3}{|c|}{$0 . / 1$. Dyn. } & \multicolumn{4}{|c|}{ 3. Dyn. } \\
\hline & & rot & schw & $\begin{array}{l}\text { rotu } \\
\text { schw }\end{array}$ & rot & gelb & grün & schw \\
\hline Q 1 & 』 & 1390 & & & & & . & \\
\hline Q 2 & A & & & & & 3372 & & \\
\hline Q 3 & $\square$ & & & & & $3371-72$ & & \\
\hline R 4 & $\stackrel{A}{ }$ & & & $646^{63}$ & & & $3372^{64}$ & \\
\hline R 8 & 7 & 1126 & & & & & & \\
\hline $\mathrm{S} 12$ & त्राता & 1390 & & & & & & \\
\hline S 20 & 8 & & & $1390^{65}$ & & & & \\
\hline S 29 & П & $\begin{array}{l}646 \\
989 a \\
990-1 \\
993-5 \\
1125 \\
1126 \\
1390 \\
1564 \\
\end{array}$ & & & $\begin{array}{l}3264^{66} \\
3371-2\end{array}$ & & & \\
\hline S 34 & $f$ & & $1564^{67}$ & & & & & \\
\hline S 42 & 8 & & $\begin{array}{l}646 \\
989 a \\
990-2 \\
994-5 \\
\end{array}$ & & & & & \\
\hline s 2 & 1 & 1564 & & & & & & \\
\hline $\mathrm{s} 2^{\mathrm{v}}$ & 1 & 1564 & & & & & & \\
\hline s 29 & 01 & 1564 & & & & & & \\
\hline s 35 & $\nabla$ & & 1564 & & & & & \\
\hline s 36 & $\square$ & & 1564 & & & & & \\
\hline T 4 & क & & & $\begin{array}{l}1117- \\
22^{68} \\
\end{array}$ & & & & \\
\hline T 7 & $a$ & & 1677 & $\begin{array}{l}990^{69} \\
1390^{70} \\
\end{array}$ & & & & \\
\hline
\end{tabular}

${ }^{63}$ Rote Binsenmatte, schwarzes Brot.

${ }^{64}$ Die Binsenmatte ist grün, zu dem Brot liegen keine Angaben vor (vgl. Murray, Saqqara Mastabas, Taf. 44).

${ }^{65}$ Zum Teil schwarze „Einsprengsel“ zu erkennen.

${ }^{66}$ Quibell, Hesy, 36.

${ }^{67} \mathrm{Da}$ der Kontext unsicher ist, könnte es sich auch um ein anderes Zeichen (z. B. S 20) handeln.

${ }^{68}$ Roter Keulenkopf, rotes Band, schwarzer Griff.

${ }^{69}$ Rote Klinge, schwarzer Schaft.

${ }^{70}$ Schwarze Klinge, roter Schaft. 


\begin{tabular}{|c|c|c|c|c|c|c|c|c|}
\hline \multirow[b]{2}{*}{$\begin{array}{c}\text { Zeichen- } \\
\text { Nr. }\end{array}$} & \multirow[b]{2}{*}{ Zeichen } & \multicolumn{3}{|c|}{ 0./1. Dyn. } & \multicolumn{4}{|c|}{ 3. Dyn. } \\
\hline & & rot & schw & $\begin{array}{l}\text { rotu } \\
\text { schw }\end{array}$ & rot & gelb & grün & schw \\
\hline T 8 & กิ & 1677 & & & $3371-2$ & & & \\
\hline Т 11 & $\hookleftarrow$ & & & & & & & $3372^{71}$ \\
\hline Т 15 & $\geqslant$ & & 1390 & & & & & \\
\hline Т 18 & 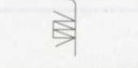 & 1677 & & & & & & \\
\hline Т 28 & 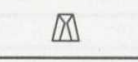 & & & & & & & $3372^{72}$ \\
\hline Т 33 & -8 & & & & 3371 & & & \\
\hline $\mathrm{U} 1$ & 3 & & 1390 & & & & & \\
\hline $\mathrm{U} 6$ & A & 1390 & & & $3371-2$ & & & \\
\hline u 9 & 四 & 1390 & & & & & & \\
\hline V 1 & p & 645 & & & & & & \\
\hline V 6 & $\gamma$ & & $1126^{73}$ & & & & & \\
\hline $\mathrm{V} 12^{\mathrm{v}}$ & $\alpha$ & $\begin{array}{l}\text { vgl. m } 2 \\
\text { (vielfach) } \\
u \mathrm{~V} 12^{\mathrm{v}}\end{array}$ & & & & & & \\
\hline V 20 & $\cap$ & & $\begin{array}{l}989 a \\
1125\end{array}$ & & & & & \\
\hline V 24 & \{ & & & & & 3372 & & \\
\hline V 28 & 8 & $\begin{array}{l}1126 \\
1390 \\
1677 \\
\end{array}$ & & & & & & \\
\hline V 30 & $\varpi$ & $\begin{array}{l}\text { vgl. } \\
\text { G } 16\end{array}$ & & & & & & \\
\hline V 33 & 8 & & & & & & & $3372^{74}$ \\
\hline v 3 & $n$ & & & & & & & $\begin{array}{l}\text { vgl. } \\
\text { v } 3 u \\
\text { W } 24\end{array}$ \\
\hline v 6 & $\infty$ & 645 & & & & & & \\
\hline v 7 & Пग & & & $1125^{75}$ & & & & \\
\hline
\end{tabular}

${ }^{71}$ Die Federn des Pfeiles sind schwarz.

${ }^{72}$ Außen schwarz, innen rot.

${ }^{73} \mathrm{Ob}$ es sich um V 6 handelt, ist unsicher.

${ }^{74}$ Schwarzer Sack, dessen oberer Teil rot gemalt ist.

${ }^{75}$ Roter Sack, am oberen Rand schwarze Punkte. 


\begin{tabular}{|c|c|c|c|c|c|c|c|c|}
\hline \multirow[b]{2}{*}{$\begin{array}{c}\text { Zeichen- } \\
\text { Nr. }\end{array}$} & \multirow[b]{2}{*}{ Zeichen } & \multicolumn{3}{|c|}{ 0./1. Dyn. } & \multicolumn{4}{|c|}{ 3. Dyn. } \\
\hline & & rot & schw & $\begin{array}{l}\text { rotu } \\
\text { schw }\end{array}$ & rot & gelb & grün & schw \\
\hline W 2 & म & $\begin{array}{l}645 \\
995 \\
\end{array}$ & & & $3264^{76}$ & & & \\
\hline W 10 & $\square$ & & & & & & & $\begin{array}{l}\text { vgl. } \\
\text { w } 5 u \\
\text { W } 10 \\
\end{array}$ \\
\hline W 12 & 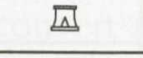 & & & & $3371-2$ & & & \\
\hline W 17 & 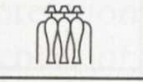 & & & $1390^{77}$ & & & & \\
\hline W 24 & 8 & $989 a^{78}$ & $\begin{array}{l}1125 \\
1390 \\
1677 \\
\text { vgl. } \\
\text { W } 25 \\
\end{array}$ & & & & & $\begin{array}{l}3264^{79} \\
3372 \\
\text { vgl. } \\
\text { v } 3 u \\
\text { W } 24\end{array}$ \\
\hline W 25 & 1 & & & $1390^{80}$ & & & & \\
\hline w 2 & O & & & $1125^{81}$ & & & & \\
\hline w 3 & D & & & & $3264^{82}$ & & & \\
\hline w 5 & $\square$ & & & & & & & $\begin{array}{l}\text { vgl. } \\
\text { w } 5 u \\
\text { W } 10 \\
\end{array}$ \\
\hline w 10 & $\ominus$ & & & & & & & $3264^{83}$ \\
\hline w 17 & $\theta$ & & & & & $3371^{84}$ & & \\
\hline w 30 & $8\}$ & 96 & & & & & & \\
\hline w 35 & $\circlearrowright$ & $\begin{array}{l}989 a \\
990 \\
994 \\
\end{array}$ & & & & & & \\
\hline X 1 & D & & $\begin{array}{l}1125 \\
1390 \\
1677 \\
\end{array}$ & & & & & $\begin{array}{l}3264^{85} \\
3371-2\end{array}$ \\
\hline y 1 & $\infty$ & & & & & $3372^{86}$ & & \\
\hline
\end{tabular}

${ }^{76}$ Oberer Teil rot und weiß gesprenkelt; unterer Teil nicht bestimmbar.

${ }^{77}$ Schwarze Krüge, rotes Gestell.

${ }^{78}$ In $\zeta_{\text {; }}$ eventuell handelt es sich um ein vom schwarzen $m w$-Topf verschiedenes Zeichen.

${ }^{79}$ Quibell, Hesy, Taf. 21.

${ }^{80}$ Rote Beine, schwarzer $n w$-Topf.

${ }^{81}$ Rotes Gefäß, oberer Rand zum Teil schwarz; roter Verschluß.

${ }^{82}$ Oberer Teil rot und weiß gesprenkelt; unterer Teil nicht bestimmbar.

${ }^{83}$ Unterer Teil schwarz und weiß, oberer Teil rot und weiß gesprenkelt.

${ }^{84}$ Gelbes Netz.

${ }^{85}$ Quibell, Hesy, 36, Taf. 10, 18, 21.

${ }^{86}$ Gelb mit schwarzen Linien. 


\begin{tabular}{|c|c|c|c|c|c|c|c|c|}
\hline \multirow[b]{2}{*}{$\begin{array}{l}\text { Zeichen- } \\
\text { Nr. }\end{array}$} & \multirow[b]{2}{*}{ Zeichen } & \multicolumn{3}{|c|}{ 0./1. Dyn. } & \multicolumn{4}{|c|}{ 3. Dyn. } \\
\hline & & rot & schw & $\begin{array}{l}\text { rotu } \\
\text { schw }\end{array}$ & rot & gelb & grün & schw \\
\hline Z 1 & 1 & & $\begin{array}{l}989 \\
1125\end{array}$ & & & & & \\
\hline Aa 1 & 首 & & & & & $3371-2$ & & \\
\hline Aa 7 & 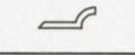 & 1390 & & & & & & \\
\hline Aa 14 & $\simeq$ & & & & 3372 & & & \\
\hline Aa 28 & \{ & & 646 & & & & & \\
\hline aa 39 & $\Delta$ & & 645 & & & & & \\
\hline $\begin{array}{l}\mathrm{m} 2 \\
\text { (viel- } \\
\text { fach) } \\
\mathrm{V} 12^{\mathrm{V}}\end{array}$ & H्र & & $1125^{87}$ & & & & & \\
\hline $\begin{array}{l}\text { v } 3 u \\
\text { W } 24\end{array}$ & 8 & & & & & & & $3264^{88}$ \\
\hline $\begin{array}{l}\text { (w } 5 u \\
\text { W } 10 \text { ) }\end{array}$ & $\square$ & & & & & & & 3371 \\
\hline
\end{tabular}

\section{LITERATUR - UND ABKÜRZUNGSVERZEICHNIS}

Baines, Color Terminology and Color Classification: John Baines, Color Terminology and Color Classification: Ancient Egyptian Color Terminology and Polychromy, in: American Anthropologist 87, 1985, 282-297.

Emery, Hemaka: Walter B. Emery, The Tomb of Hemaka. Cairo 1938 (Excav. Saqq.).

Emery, Tombs of the First Dynasty: Walter B. Emery, Great Tombs of the First Dynasty, 3 Bde. Cairo - London 1949-58 (Excav. Saqq.).

Gardiner, Egyptian Grammar: Alan Gardiner, Egyptian Grammar. ${ }^{3}$ London 1957.

Godron, Horus Den: Gérard Godron, Études sur l'Horus Den et quelques problèmes de l'Égypte archaique. Genève 1990 (Cahiers d'Orientalisme 19).

Kahl, System: Jochem Kahl, Das System der ägyptischen Hieroglyphenschrift in der 0.-3. Dynastie. Wiesbaden 1994 (GOF IV, 29).

Kaplony, Inschriften Supplement: Peter Kaplony, Die Inschriften der ägyptischen Frühzeit. Supplement. Wiesbaden 1964 (ÄA 9).

Murray, Saqqara Mastabas: Margaret A. Murray, Saqqara Mastabas I. London 1905 (ERA 10).

Petrie, Royal Tombs: W. M. Flinders Petrie, The Royal Tombs of the First Dynasty. Part I. London 1900 (EEF 18); The Royal Tombs of the Earliest Dynasties. Part II. London 1901 (EEF 21).

Quibell, Archaic Mastabas: James Edward Quibell, Archaic Mastabas. Le Caire 1923 (Excav. Saqq. 1912-14).

Quibell, Hesy: J. E. Quibell, The Tomb of Hesy. Le Caire 1913 (Excav. Saqq. 1911-12).

Schenkel, Farben: Wolfgang Schenkel, Die Farben in ägyptischer Kunst und Sprache, in: ZÄS 88, 1963, $131-147$.

Smith, Sculpture and Painting: William Stevenson Smith, A History of Egyptian Sculpture and Painting in the Old Kingdom. ${ }^{2}$ London 1949.

Staehelin, Farben: Elisabeth Staehelin, Zu den Farben der Hieroglyphen, in: Erik Hornung, Zwei ramessidische Königsgräber: Ramses IV. und Ramses VII. Mainz 1990 (Theben 11) 101-119.

Thomas, Discovery: Nancy Thomas, The American Discovery of Ancient Egypt. Los Angeles 1995.

${ }^{87}$ Rote Schnur, schwarze Feigen.

${ }^{88}$ Quibell, Hesy, Taf. 21. 